\title{
A Comparative Study of Wavelet and Curvelet Transform for Image Denoising
}

\author{
Miss Monika shukla ${ }^{1}$, Dr.Soni changlani ${ }^{2}$ \\ ${ }^{1}$ (Department.of Electronics \& telecommunication;Lakshmi Narain College of Technology \& Science,BHOPAL) \\ ${ }^{2}$ (Head of Department.of Electronics \& telecommunication;Lakshmi Narain College of Technology \& \\ Science, BHOPAL)
}

\begin{abstract}
This paper describes a comparison of the discriminating power of the various multiresolution based thresholding techniques i.e., Wavelet, curve let for image denoising.Curvelet transform offer exact reconstruction, stability against perturbation, ease of implementation and low computational complexity. We propose to employ curve let for facial feature extraction and perform a thorough comparison against wavelet transform; especially, the orientation of curve let is analysed. Experiments show that for expression changes, the small scale coefficients of curve let transform are robust, though the large scale coefficients of both transform are likely influenced. The reason behind the advantages of curvelet lies in its abilities of sparse representation that are critical for compression, estimation of images which are denoised and its inverse problems, thus the experiments and theoretical analysis coincide .
\end{abstract}

Keywords: Curvelet transform, Face recognition, Feature extraction, Sparse representation Thresholding rules, Wavelet transform.

\section{Introduction}

Image denoising refers to the recovery of a digital image that has been contaminated by Additive white Gaussian Noise (AWGN). AWGN is a channel model in which the only impairment to communication is a linear addition of wideband or white noise with a constant spectral density(expressed as watts/ $\mathrm{Hz}$ of bandwidth) and a Gaussian distribution of amplitude.

On a daily basis, hospitals are witnessing a large inflow of digital medical images and related clinical data. The main hindrance is that an image gets often corrupted by noise in its acquisition and transmission [1]. Image denoising is one of the classical problems in digital image processing, and has been studied for nearly half a century due to its important role as a pre - processing step in various electronic imaging applications. Its main aim is to recover the best estimate of the original image from its noisy versions [2]. Wavelet transform enable us to represent signals with a high degree of scarcity. This is the principle behind a non-linear wavelet based signal estimation technique known as wavelet denoising. In this paper we explore wavelet denoising of images using several thresholding techniques such as SURE SHRINK, VISU SHRINK and BAYES SHRINK. Further, we use a Gaussian based model to perform combined denoising and compression for natural images and compare the performance of wave transform methods [3].

In this paper, we also describe approximate of new mathematical transforms, namely as curvelet transform for image denoising [4]. Our implementations offer exact reconstruction, stability against perturbations, ease of implementations and low computational complexity. A central tool is Fourier domain computation of an approximate digital random transform. In a curvelet transform, we will use sparsity and its applications [5].

In the past, we have proposed a work on novel image denoising method which is based on DCT basis and sparse representation [6].

To achieve a good performance in these aspects, a denoising procedure should adopt to image discontinuities. Therefor, a comparative study on mammographic image denoising technique using wavelet, and curvelet transform [7]. Therefore, multi resolution analysis [8] is preferred to enhance the image originality. The transform domain denoising typically assumes that the true image can be well approximated by a linear combination of few basis elements. That is, the image is sparsely represented in the transform domain. Hence, by preserving the few high magnitude transform coefficients that convey mostly the original image property and discarding the rest which are mainly due to noise, the original image can be effectively estimated [9]. The sparsity of the representation are critical for compression of images, estimation of images and its inverse problems. A sparse representation for images with geometrical structure depends on both the transform and the original image property. In the recent years, there has been a fair amount of research on various denoising methods like wavelet, curvelet contourlet and various other multi resolution analysis tools. Expectation Maximization (EM) algorithm introduced by Figueirodo and Robert [10] for image restoration based on penalized livelihood formulized in wavelet domain. State-of-art Gaussian Scale Mixture (GSM) algorithms 
employs modelling of images according to the activity within neighbourhoods of wavelet coefficients and attaching coefficients heavily in inactive regions [11]. Coif man and Donoho [12] pioneered in wavelet thresholding pointed out that wavelet algorithm exhibits visual artefacts'. Curvelet transform is a multi scale transform with strong directional character in which elements are highly anisotropic at fine Scales. The developing theory of curvelets predict that, in recovering images which are smooth away from edges, curvelets obtain smaller asymptotic mean square error of reconstruction than wavelet methods [13].

\section{Multiresolution Techniques:}

An image can be represented at different scales by multi resolution analysis. It preserves an image according to certain levels of resolution or blurring in images and also improves the effectiveness of any diagnosis system [14].

\subsection{Wavelet:}

Wavelet transform can achieve good scarcity for spatially localized details, such as edges and singularities. For typical natural images, most of the wavelet coefficients have very small magnitudes, except for a few large ones that represent high frequency features of the image such as edges. The DWT (Discrete wavelet transforms) is identical to a hierarchical sub band system. In DWT,the original image is transformed into four pieces which is normally labelled as $\mathrm{A} 1, \mathrm{H} 1, \mathrm{~V} 1$ and $\mathrm{D} 1$ as the schematic depicted in fig.1.The A1 sub-band called the approximation, can be further decomposed into four sub-bands. The remaining bands are called detailed components. To obtain the next level of decomposition, sub-band A1 is further decomposed.

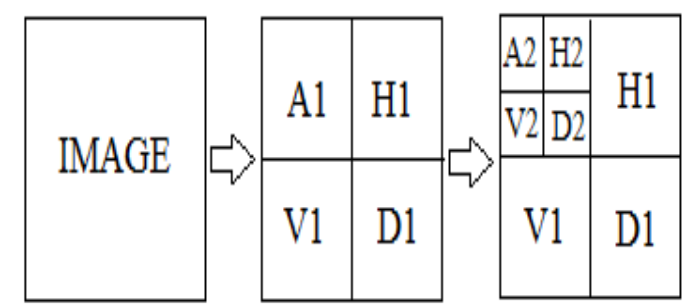

Figure 1. DWT based Wavelet decomposition to various levels

Many wavelet's are needed to represent an edege(number depends on the lengh of the edge,not the smoothness).In this,m-term approximation error would be occur.

\section{ORIGINAL:}

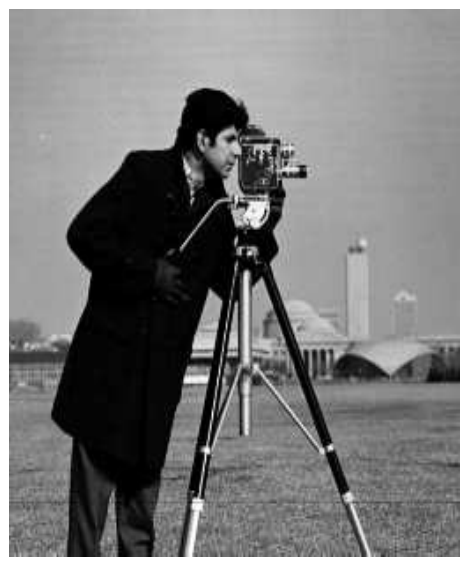

( || $\left.\mathbf{f}-\mathbf{f}_{\mathrm{m}}||{ }_{2}\right)^{2} \approx \mathbf{m}^{-1}$ 1\% OF WAVELET COEFFS: $10 \%$ OF WAVELET COEFFS:
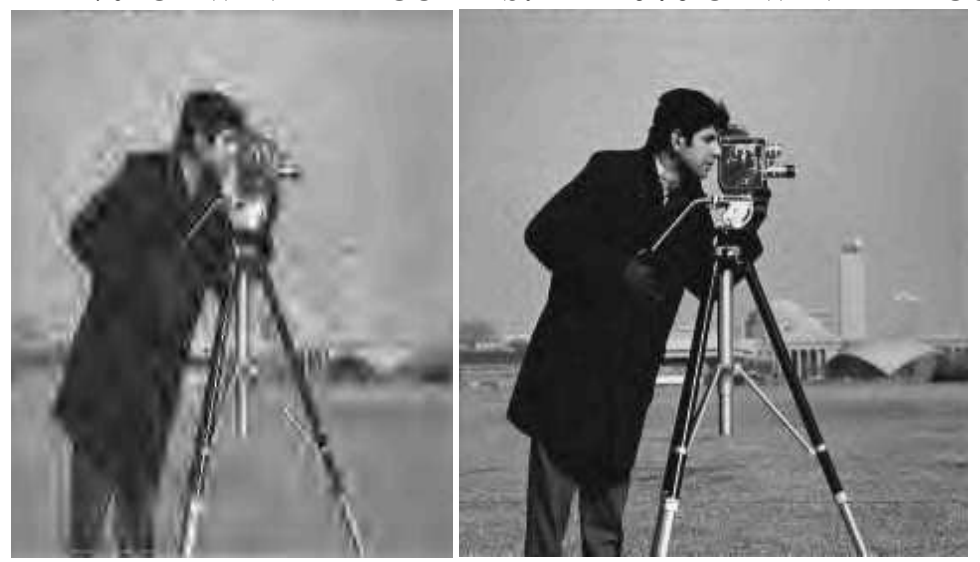

Wavelets and its Geometry: The basis function of wavelets is isotropic. They cannot "adapt" to geometrical structure. In this we need more refined scaling concepts. 

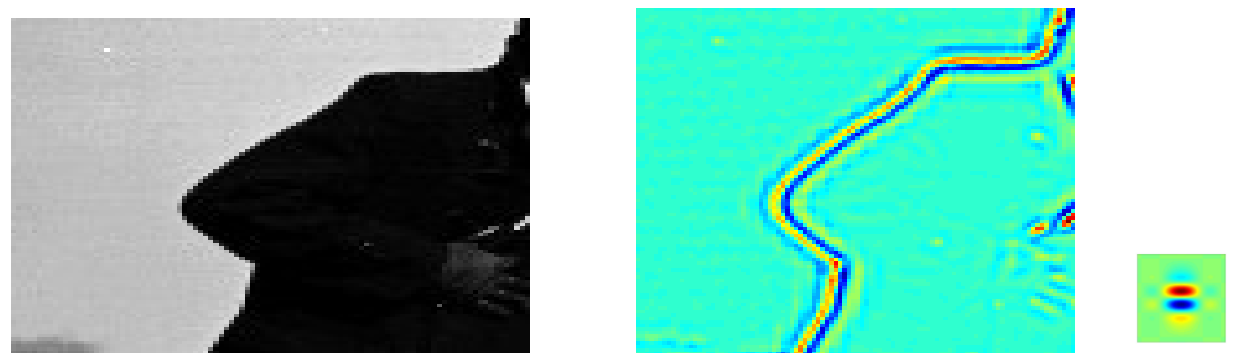

\subsection{Curvelet:}

Curvelets are a non-adaptive technique for multi-scale object representation. Being an extension of the wavelet concepts, they are becoming popular in similar fields, namely in image processing and scientific computing.Curvelet transform is a multi-scale geometric wavelet transforms, can represent edges and curves singularities much more efficiently than traditional wavelet.Curvelet combines multiscale analysis and geometrical ideas to achieve the optimal rate of convergence by simple thresholding.Multi-scale decomposition captures point discontinuities into linear structures.Curvelets in addition to a variable width have a variable length and so a variable anisotropy. The length and width of a curvelet at fine scale due to its directional characteristics is related by the parabolic scaling law:

\section{Width $\sim(\text { length })^{2}$}

Curvelets partition the frequency plan into dyadic coronae that are sub partitioned into angular wedges displaying the parabolic aspect ratio as shown in fig.2. Curvelets at scale $2^{-\mathrm{k}}$, are of rapid decay away from a 'ridge' of length $2^{-k / 2}$ and width $2^{-k}$ and this ridge is the effective support. The discrete translation of curvelet transform is achieved using wrapping algorithm[15]. The curvelet coefficients $\mathrm{C}_{\mathrm{k}}$ for each scale and angle is defined in Fourier domain by

$$
C_{k}(r, \theta)=2^{-3 k / 4} R\left(2^{-k} r\right) A\left(2^{(k / 2)} / 2 \pi . \theta\right)
$$

Where $C_{k}$ in this equation represents polar wedge supported by the radial(R) and angular (A) windows.

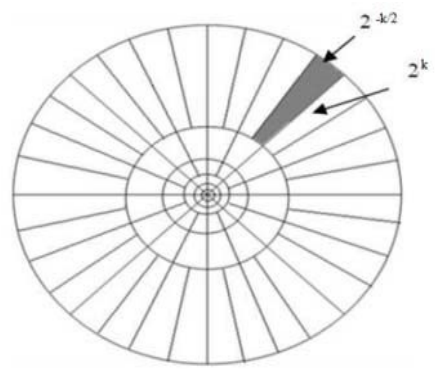

Figure 2. Curvelets in Frequency Domain

Digital Curvelet Transform can be implemented in two ways (FDCT via USFFT and FDCT via wrapping),which differ by spatial grid used to translate curvelets at each scale and angle.[16].

\section{Proposed Work}

In this paper, we report initial efforts at image denoising based on a recently introduced family of transforms- Wavelet transform and Curvelet transform. In this paper, we compare the results from wavelet transform and curvelet transform and we will see which transform is better for the image denoising. Our main objective is to decrease a mean square error (MSE) and to increase a peak signal to noise ratio (PSNR) in db.by adding a white noise like Gaussian noise, Poisson noise and Speckle noise. During this configuration, we will use Threshold estimator like heursure, rigrsure, sqtwolog, and minimaxi. We can adjust decomposition level from 1 to 5 and we use Thresholding [17].Thresholding is the simplest method of image segmentation. From a greyscale image, thresholding can be used to create binary images.Thresholding is a simple non-linear technique, which operates on one wavelet coefficient at a time. In its most basic form, each coefficient is threshold by comparing against threshold.If the coefficient is smaller than threshold, set to zero, otherwise it is kept or modified. On replacing the small noisy coefficients by zero and inverse wavelet transform. In both case (Soft thresholding and Hard thresholding) the coefficients that are below a certain threshold are set to zero.

In hard thresholding,the remaining coefficients are left unchanged. In soft thresholding, the magnitudes of the coefficients above threshold are reduced by an amount equal to the value of the threshold. In both cases, 
each wavelet coefficient is multiplied by a given shrinkage factor, which is a function of the magnitude of the coefficient.

In our thesis, we will use a curvelet transform as well as wavelet transform for removing a additive noise which is present in our images and we will also compare between both the techniques i.e.,Curvelet transform and Wavelet transform which we are using \& we'll see which one is better for image denoising.

\section{Materials \& Methods}

Image from MIAS database was denoised using wavelet and curve let transforms. Various types of noise like the Random noise, Gaussian noise, Salt\&Pepper and speckle noise were added to this image

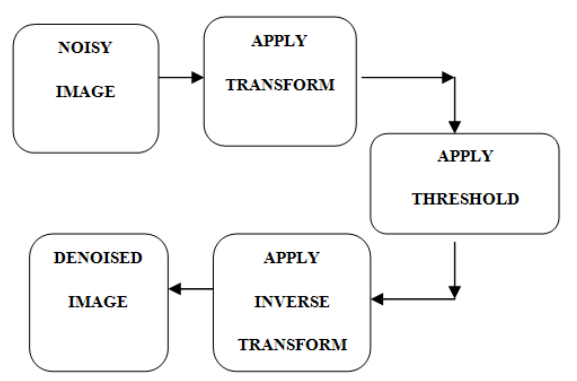

\section{- Algorithm}

Denoising procedure followed here is performed by taking wavelet/curvelet transform of the noisy image Random, Salt and Pepper, Poisson, Speckle and Gaussian noises) and then applying hard thresholding technique to eliminate noisy coefficients. The algorithm is as follows:

Step1: Computation of threshold

Step2: Apply wavelet/curvelet/contourlet transform to image

Step3: Apply computed thresholds on noisy image

Step4: Apply inverse transform on the noisy image to transform image from transform domain to spatial domain.

\section{Experimental Results}

The Experiment was done on several natural images like lena, Barbara,,baboon,cameraman etc.using multiple denoising procedures for several noises. In our experiment, we have considered a image of A cricketer Mahendra Singh dhoni.In this image we have used a different additive noises like Gaussian noise, poisson noise, and speckle noise with different noise levels $\sigma=10,15,20,25,30,35$ etc. And before adding a noise,,mean value is always be 0 .

\begin{tabular}{|l|l|l|l|}
\hline NOISES & $\begin{array}{l}\text { NOISY } \\
\text { IMAGES } \\
\text { PSNR/db }\end{array}$ & $\begin{array}{l}\text { WAVELET } \\
\text { PSNR/db }\end{array}$ & $\begin{array}{l}\text { CURVELET } \\
\text { PSNR/db }\end{array}$ \\
\hline Poisson & 27.7344 & 27.0602 & 33.8397 \\
\hline Gaussian & 24.9825 & 26.2889 & 32.4896 \\
\hline Speckle & 30.2455 & 27.4944 & 34.8447 \\
\hline
\end{tabular}

Table.A: Comparison Of Wavelet And Curvelet With Different Noise In Psnr.

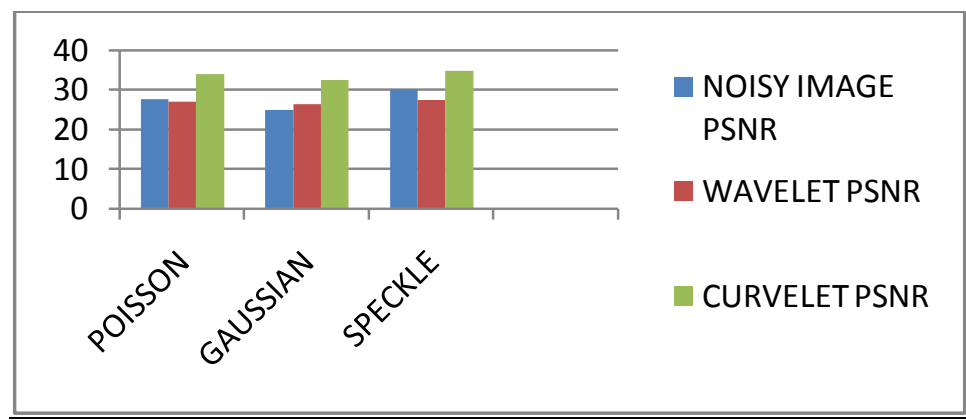

Fig.A: Graph indicating comparative results of the PSNR values of wavelet and curvelet based thresholding for image denoising . 
Table A. shows the comparison of wavelet and curvelet with different noises and we measures the peak signal to noise ratio(in $\mathrm{Db}$ ) and Fig.A shows a graph which indicates a comparative results of the PSNR values of wavelet and curvelet based thresholding(soft/hard) for image denoising and there is, we apply a different types of threshold estimator like rigrsure,heursure,sqtwolog,mini-maxi. And different decomposition levels like 1,2,3,4,5 \& so on

\begin{tabular}{|l|l|l|l|}
\hline NOISES & $\begin{array}{l}\text { NOISY } \\
\text { IMAGES } \\
\text { MSE }\end{array}$ & $\begin{array}{l}\text { WAVELET } \\
\text { MSE }\end{array}$ & $\begin{array}{l}\text { CURVELET } \\
\text { MSE }\end{array}$ \\
\hline Poisson & 109.5562 & 127.9571 & 26.8605 \\
\hline Gaussian & 207.5685 & 152.8252 & 36.6541 \\
\hline Speckle & 61.4507 & 115.7913 & 23.3111 \\
\hline & & & \\
\hline
\end{tabular}

Table.B: Comparison Of Wavelet And Curvelet With Different Noise In Mse.

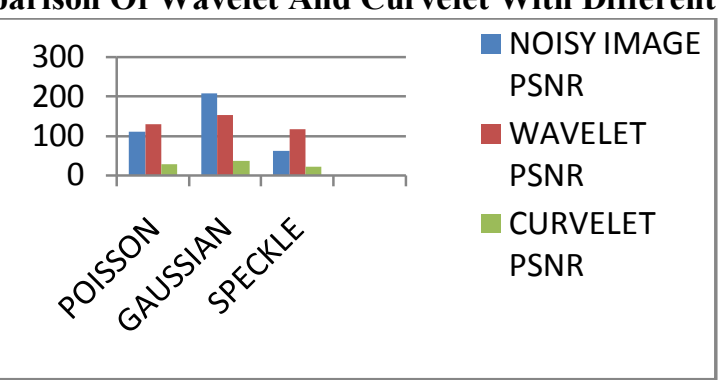

Fig.B: Graph indicating comparative results o the MSE values of wavelet and curvelet based thresholding for image denoising

Table B. shows the comparison of wavelet and curvelet with different noises. we measures the mean square error(MSE) and Fig.B shows a graph which indicates a comparative results of the MSE values of wavelet and curvelet based thresholding(soft/hard) for image denoising and there is, we apply a different types of threshold estimator like rigrsure,heursure,sqtwolog,mini-maxi.and different decomposition levels like 1,2,3,4,5\& so on.

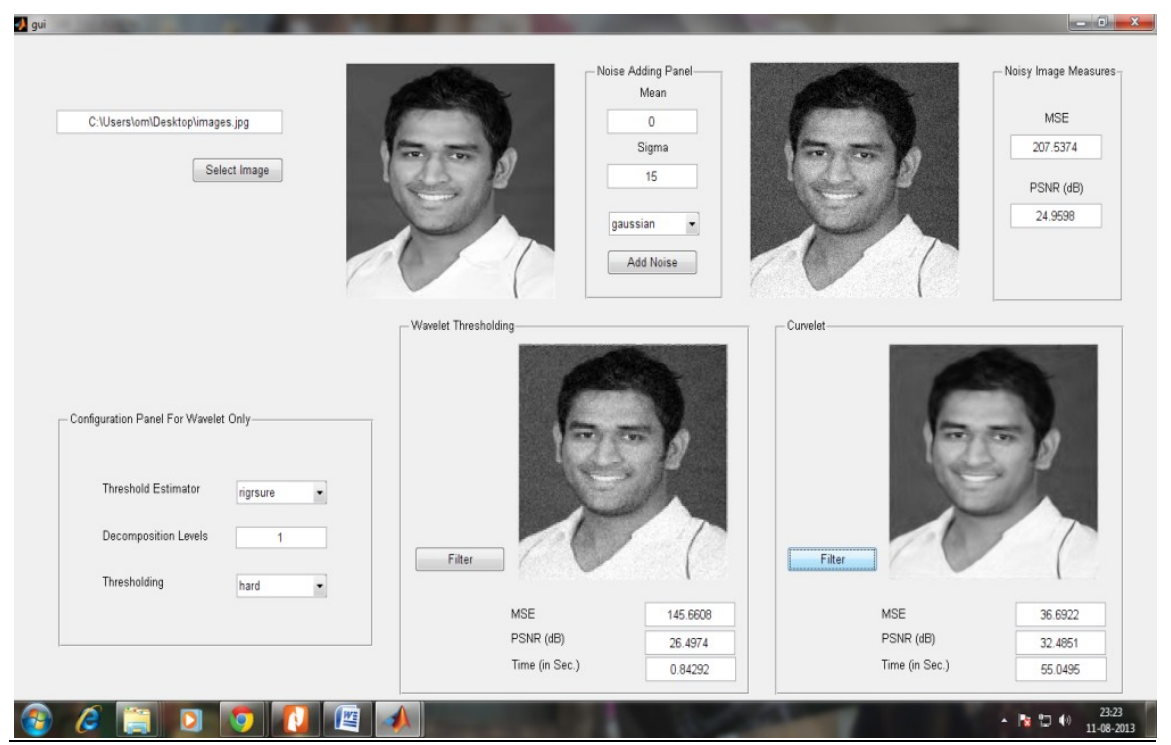

\section{Conclusion}

The comparison of wavelet transform and curvelet transform technique is rather a new approach, and it has a big advantage over the other techniques that it less distorts spectral characteristics of the image denoising. The experimental results show that the curvelet transform gives better results/performance than wavelet transform method. The primary goal of noise reduction is to remove the noise without losing much detail contained in an image. To achieve this goal, we make use of a mathematical function known as the wavelet transform to localize an image into different frequency components or useful sub bands and effectively reduce the noise in the sub bands according to the local statistics within the bands. The main advantages of the wavelet transform \& curvelet transform is that the Image fidelity after reconstruction is visually losseless.Image 
processing task, both as a process itself and as a image denoising is an important component in other processes. Very many ways to denoise an image or a set of data exists. The main properties of a good image denoising model is that it will remove a noise while preserving edges. Traditionally,linear models have been used. One common approach is to use a Gaussian Filter or equivalently solving the heat equation with the noisy image as input-data i.e., a linear, $2^{\text {nd }}$ order PDE-model.

One big advantage of linear noise removal models is the speed,but a drawback of the linear models is that they are not able to preserve edges in a good manner. This paper presents a review of some significant work in the area of image denoising.After a brief introduction,some popular approaches are classified into different groups and an overview of various algorithms and analysis is provided.Insights \& potential future trends in the area of denoising are also discussed.

\section{Acknowledgement}

The Author would like to thanks The Rajiv Gandhi Proudyogiki Vishwavidyalaya,,BHOPAL. for its generous support ,and The Lakshmi Narain College Of Technology and Science,BHOPAL.for their hospitality, during my academic period 2011-2013. She wishes to thanks Dr.Soni Changlani and Mr. Trijender singh for their help and encouragement.

\section{References}

[1] R.C.Gonzalez and R.E. woods, digital image processing $2^{\text {nd }}$ edition;pearson education.

[2] D.Ghandurai and V.sadasivam,"an effective adaptive thresholding technique for wavelet based image denoising."World academy of science,engg.\&technology,2008.

[3] Raghuram Rangarajan,Ramji venkataramanan,siddharth shah,Image denoising using wavelet;Dec 16,2002.

[4] Jean-luc starck,Emmanuel J.candes,and David L. Donoho; The curvelet transform for image denoising;IEEE translations on image processing,vol.11,no.6,june 2002.

[5] Emmanuel candes;California institute of technology.

[6] Zhang fen,Xiekai;college of information \& mechanical engineering; Beijing Institute of Graphic communication,Beijing,china.

[7 ] E.Malar, Akhandaswamy,S.S.kirthana,D.Nivedhitha;Department of bio medical engg.PSG College of technology, Coimbatore,India

[8] J.L. starck,M.Elad,and D.Donoho "Redundant Multiscale transforms and their application for Morphological component separation," Advances in imaging and Electron Physics,Vol.132,pp.287-348,2004.

[9] H.Rabbani, “ Image denoising in steerable pyramid domain based on a local laplace prior, " pattern recognition' , vol.42,no.9,September 2009,pp.2181-2193.

[10] M.Figeiredo and R.Nowak, “ An EM algorithm for wavelet based image restoration,”.IEEE Transaction on Image Processing,vol.12.No.8,pp 906-916,August 2003

[11] J.Portilla,V.Strela,M.J wain wright and E.P Simon celli, "Image denoising using Gaussian scale mixtures in the wavelet domain, "IEEE Transaction on Image Processing."

[12] R.R.Coifman and D.L. Donoho, "Translation - invariant denoising."

[13] E.Candes and D.Donoho, "New tight frames of curvelets and optimal representations of objects with piecewise singularities".

[14] S.Liu,C.F.Babbs and E.J. Delp, “Multiresolution detection of speculated lesions in digital mammograms”,IEEE transactions on Image Processing.

[15] E.Malar,A kandaswamy;PSG College of technology,Coimbatore;India.

[16] A patil \& Jyoti Singhai, COE,Malegaon,Pune;India.

[17] From Wikipedia. The free encyclopedia,Gonzalez,Rafael C.\& Woods, Richard E(2002).Thresholding in digital image processing, Pearson Edition. 